\title{
Chronic congestive heart failure - a new therapeutic choice
}

\author{
John D. Parker MD, Heather J. Ross MD
}

$\mathrm{T}$ he 2014 PARADIGM-HF (Prospective Comparison of ARNI with ACEI to Determine Impact on Global Mortality and Morbidity in Heart Failure Trial) study ${ }^{1}$ compared the effectiveness of a novel combination of neprilysin (an endopeptidase) inhibitor sacubitril and angiotensin II receptor antagonist valsartan (sacubitril/ valsartan) with the angiotensin-converting-enzyme (ACE) inhibitor enalapril in 8442 patients who had heart failure (identified as New York Heart Association classes II-IV) with reduced ejection fraction. The trial results were striking. Sacubitril/ valsartan was associated with a $20 \%$ reduction in death due to cardiovascular causes, as well as a $21 \%$ reduction in admissions to hospital for congestive heart failure. After an expedited review process, sacubitril/valsartan was recently approved by Health Canada to treat heart failure with reduced ejection fraction. What are the implications of these findings for clinicians in Canada?

Chronic congestive heart failure affects more than 500000 Canadians. $^{2}$ Treatment for congestive heart failure secondary to left ventricular systolic dysfunction (heart failure with reduced ejection fraction), which represents about $50 \%$ of the total burden of congestive heart failure, ${ }^{3}$ has improved dramatically over the past 30 years. ${ }^{4}$ Treatments that have been shown to improve clinical outcome include ACE inhibitors, angiotensin II receptor antagonists, $\beta$-blockers, aldosterone receptor antagonists and sinus node inhibiting agents. Many patients are also treated with loop diuretics. In addition, cardiac resynchronization and device treatments may improve outcomes in appropriately selected patients. A few patients may also have atrial fibrillation that may or may not require specific pharmacotherapy. Furthermore, there are often comorbidities that warrant additional pharmacotherapy. Therefore, the modern management of heart failure presents a complex therapeutic challenge that requires frequent clinical interactions over time, with surveillance of both clinical and biochemical responses to treatment. Historically, as new treatments for congestive heart failure were developed, they were added to previous proven treatments for patients with appropriate clinical characteristics.

In anticipation of the approval of sacubitril/ valsartan, the 2014 Canadian Cardiovascular
Society Heart Failure Management Guidelines Focus Update suggested that patients with mildto-moderate heart failure would be eligible to start this treatment. ${ }^{5}$ Importantly, the new drug would replace treatment with traditional ACE inhibitors or angiotensin receptor antagonists, because beneficial effects of sacubitril/valsartan on morbidity and mortality were observed when the drug was administered instead of (not in addition to) enalapril. Many patients in Canada meet the inclusion criteria used in the PARADIGM-HF study. ${ }^{1}$ The availability of this new drug to treat heart failure with reduced ejection fraction gives physicians an important new therapeutic choice. However, withdrawing patients with symptomatic heart failure with reduced ejection fraction from current treatment with ACE inhibitors or angiotensin receptor blockers and starting treatment with sacubitril/valsartan presents a challenge.

The PARADIGM-HF study involved a group of stable patients with carefully defined characteristics, who were already known to tolerate the experimental treatment used in the study. Patients who met the inclusion criteria in this study were those with mild-to-moderate heart failure and a left ventricular ejection fraction of less than $35 \%$, and who were undergoing stable medical treatment for at least one month. Patients with heart failure in the setting of acute coronary syndrome, recent onset heart failure and those with acute decompensated

\section{KEY POINTS}

- Current treatment for congestive heart failure with reduced ejection fraction includes the use of angiotensin-converting-enzyme (ACE) inhibitors and/or angiotensin II receptor antagonists, $\beta$-blockers and mineralocorticoid receptor antagonists.

- A new agent, a combination of valsartan and the neprilysin inhibitor sacubitril (valsartan/sacubitril) has been shown to improve morbidity and mortality in patients with stable congestive heart failure (identified as New York Heart Association classes II and III) with reduced ejection fraction.

- Before starting treatment with valsartan/sacubitril, the withdrawal of therapy with ACE inhibitors and/or angiotensin II receptor antagonists is required; ACE inhibitors must be stopped for at least 36 hours before beginning treatment with valsartan/sacubitril.

- Patient selection is important: patients who are unstable or have advanced (class IV) symptoms should not be started on this new treatment.

- Careful clinical and laboratory follow-ups of patients who have started treatment with valsartan/sacubitril are required, as some patients may experience adverse clinical events as a consequence. 
heart failure were excluded. Furthermore, before participating in the randomized phase of the trial, eligible patients went through single-blind run-in phases in which they had to show that they could tolerate enalapril (10 mg taken twice daily) for two weeks, followed by four to six weeks of treatment with sacubitril/valsartan (100 mg taken twice daily) that was subsequently increased to $200 \mathrm{mg}$ taken twice daily. Patients were then randomly assigned only if they tolerated these sequential runin phases. As such, the results of the PARADIGMHF study were observed in a group of stable patients with carefully defined characteristics who had already been shown to tolerate the experimental therapy tested in the study.

Some eligible patients may have been stable for years on their current treatment regimen with an ACE inhibitor or an angiotensin receptor antagonist. Others may have been less stable and more symptomatic, with tenuous blood pressure and marginal renal function. In many provinces (if not all), sacubitril/valsartan will not be covered initially by provincial formularies. Moreover, initiation of the new treatment in patients who may benefit will require clinical and biochemical status reviews and careful planning for the start and uptitration of treatment. Transition to the new treatment must be made cautiously, because a change in either clinical status or biochemical parameters may be observed during the transition period. Multiple clinic visits to evaluate early clinical and biochemical responses to treatment will be required, because some patients' conditions may deteriorate during the transition. In the PARADIGM-HF study, about $7 \%$ of patients had an adverse clinical event during the run-in phase with enalapril, and an additional $7 \%$ had adverse events when they started sacubitril/valsartan. ${ }^{1}$ Furthermore, because there is a risk of angioneurotic edema, ACE inhibitors must be withdrawn 36 hours before starting sacubitril/valsartan (www.novartis.ca/sites/www. novartis.ca/files/entresto_scrip_e.pdf). Therapeutic vigilance will be essential.

The recommended starting dose of sacubitril/ valsartan is $100 \mathrm{mg}$ (sacubitril [49 mg] plus valsartan [51 mg]) taken twice daily; however, in patients with marginal blood pressure, the lowest dose should be used (50 mg taken twice daily; sacubitril [24 mg] plus valsartan [26 mg]) and titrated upward, as tolerated, to higher dose ranges (www.novartis.ca/sites/www.novartis.ca/ files/entresto_scrip_e.pdf).

Some commentators have expressed concerns about changing practice based on the findings of a single trial showing clinical efficacy of this new drug. ${ }^{6}$ Such concerns, and knowing that the initiation of new treatment will require the withdrawal of well-established regimens, may cause reluctance in some practitioners to change treatment regimens for their patients. Switching treatment in patients with severe chronic heart failure will be particularly controversial, because the PARADIGM-HF trial did not include many patients with truly advanced chronic heart failure. Although the study included patients with New York Heart Association class IIIV symptoms, less than $25 \%$ of the patient population had class III heart failure and less than $1 \%$ had class IV. Health Canada approval of sacubitril/valsartan did not extend to patients with class IV heart failure, and caution about treatment with sacubitril/ valsartan in these patients until more data are available is warranted. However, patients with advanced chronic heart failure often bear a substantial symptomatic burden, frequently require admission to hospital and are at high risk of death. On the one hand, these patients may have the most to gain from an effective new therapeutic approach. On the other, there is insufficient evidence to suggest they will benefit from this new treatment, and it is possible that the risk of adverse consequences of switching treatment would be greater in this group.

In light of a new therapeutic choice for those who care for patients with chronic congestive heart failure, physicians are faced with a challenge in deciding which patients are most likely to benefit from a treatment substitution and least likely to suffer adverse sequelae. For the health care system, challenges will be presented in the form of finding time, personnel and resources to initiate and monitor therapeutic changes in an area of care already pressed to provide adequately for a complex patient population. However, for carefully selected patients, the new therapy represents an opportunity to refine and improve treatment for a high-burden disease.

\section{References}

1. McMurray JJ, Packer M, Desai AS, et al. Angiotensin-neprilysin inhibition versus enalapril in heart failure. N Engl J Med 2014; 371:993-1004.

2. Blais C, Dai S, Waters C, et al. Assessing the burden of hospitalized and community-care heart failure in Canada. Can J Cardiol 2014:30:352-8.

3. Udelson JE. Heart failure with preserved ejection fraction. Circulation 2011;124:e540-3.

4. Sacks CA, Jarcho JA, Curfman GD. Paradigm shifts in heartfailure therapy — a timeline. N Engl J Med 2014;371:989-91.

5. Moe GW, Ezekowitz JA, O'Meara E, et al. The 2014 Canadian Cardiovascular Society Heart Failure Management Guidelines Focus Update: anemia, biomarkers, and recent therapeutic trial implications. Can J Cardiol 2015;31:3-16.

6. Califf RM. LCZ696: Too good to be true? Eur Heart J 2015; 36:410-2.

Affiliations: Division of Cardiology, Department of Medicine (Parker), Mount Sinai Hospital; Division of Cardiology, Department of Medicine (Parker, Ross), University Health Network Hospitals; Lunenfeld-Tanenbaum Research Institute (Parker); Peter Munk Cardiac Centre (Parker, Ross), Toronto General Hospital, Toronto, Ont.

Contributors: Both of the authors contributed substantially to the concept of the article, drafted or revised the manuscript critically for important intellectual content, approved the final version to be published and agreed to act as guarantors of the work. 